\title{
Propelling polysulfides transformation for high-rate and long-life lithium-sulfur batteries
}

Cheng Zheng ${ }^{\mathrm{a}, \mathrm{b}}$, Shuzhang Niu ${ }^{\mathrm{a}}$, Wei Lv ${ }^{\mathrm{a}^{*}}$, Guangmin Zhou ${ }^{\mathrm{c}}, \mathrm{Jia} \mathrm{Li}^{\mathrm{a}}$, Shaoxun Fan ${ }^{\mathrm{a}}$, Yaqian Deng $^{\mathrm{a}}$, Zhengze Pan ${ }^{\mathrm{a}}$, Baohua $\mathrm{Li}^{\mathrm{a}}$, Feiyu Kang ${ }^{\mathrm{a}}$ and Quan-Hong Yang ${ }^{\mathrm{a}^{*}}$

${ }^{a}$ Engineering Laboratory for Functionalized Carbon Materials and Shenzhen Key Laboratory for Graphene-based Materials, Graduate School at Shenzhen, Tsinghua University, Shenzhen 518055, China

${ }^{\mathrm{b}}$ School of Materials and Energy, Guangdong University of Technology, Guangzhou 510006, China

${ }^{c}$ Shenyang National Laboratory for Materials Science, Institute of Metal Research, Chinese Academy of Science, Shenyang 110016, China

Corresponding authors: Tel. /Fax: +86-755-2603-6413

E-mail: 1v.wei@sz.tsinghua.edu.cn (W. Lv); yang.quanhong@sz.tsinghua.edu.cn and qhyangcn@tju.edu.cn (Q.-H. Yang)

\begin{abstract}
:
A three-dimensional (3D) hierarchical porous graphene macrostructure coupled with uniformly distributed $\alpha-\mathrm{Fe}_{2} \mathrm{O}_{3}$ nano-particles (denoted Fe-PGM) was designed as a sulfur host in a Lithiumsulfur battery, and was prepared by a hydrothermal method. In this hybrid structure, the $\alpha-\mathrm{Fe}_{2} \mathrm{O}_{3}$ nanoparticles are proved to not only strongly interact with the polysulfides, but more importantly, chemically promote their transformation to insoluble species during the charge/discharge process, working as a chemical barrier for the shuttling of the lithium
\end{abstract}


polysulfides (LiPSs). Therefore, together with 3D hierarchical porous structure facilitating fast electron/ion transfer, Fe-PGM as a sulfur host in a cathode contributes to a high rate performance $\left(565 \mathrm{mAh} \mathrm{g}^{-1}\right.$ at a high rate of $5 \mathrm{C}$ relative to $1571 \mathrm{mAh} \mathrm{g}^{-1}$ at $0.3 \mathrm{C}$ ) as well as long cyclic stability (an ultralow capacity fading rate of $0.049 \%$ per cycle over 1000 cycles was also realized at the high current rate of $5 \mathrm{C}$ ).

Keywords: Lithium-sulfur batteries, 3D $\alpha-\mathrm{Fe}_{2} \mathrm{O}_{3} /$ graphene hybrid, redox reaction, polysulfides transformation

\section{Introduction}

Lithium-sulfur ( $\mathrm{Li}-\mathrm{S}$ ) batteries have recently attracted considerable attention as promising energy storage devices due to their high theoretical energy density of $2600 \mathrm{Wh} \mathrm{kg}^{-1}$ [1-8], which is much higher than those of conventional lithium-ion batteries [9-11], as well as the low cost and environmental friendliness of sulfur. However, their practical use is greatly hindered by the low utilization of sulfur and the poor cyclic stability due to the following reasons: (i) the electrical insulation nature of sulfur and discharge products $\left(\mathrm{Li}_{2} \mathrm{~S} / \mathrm{Li}_{2} \mathrm{~S}_{2}\right)$, (ii) the large volume expansion $(\sim 80 \%)$ of sulfur upon lithiation, and (iii) the shuttle of the soluble intermediate lithium polysulfides (LiPSs) [12-16]. In order to solve these problems, conductive porous carbons have been widely used to encapsulate sulfur to improve the electronic conductivity of the cathode and restrain the shuttle of the LiPSs. Small micropores in these carbons show high efficiency to confine the LiPSs because only desolvated $\mathrm{Li}$ ions can enter and react with sulfur in these pores. However, this greatly lowers the reaction kinetics due to the slow desolvation process and low reactivity of sulfur with deficient electrolyte. Moreover, the ions diffuse slowly in the tortuous porous structures. Therefore, it is still challenging to simultaneously reach high rate performance and long cyclic stability for the carbon-sulfur hybrid cathodes for real use. 
Most recently, graphene-based assemblies have been considered as promising carbon hosts in Li-S batteries because of their excellent conductivity and high surface area [17-19]. They are favourable for the fast reaction kinetics during the charge/discharge process due to the twodimensional structure and the aggregated, easily accessible large pores. However, this structure is hard to effectively restrain the shuttling of LiPSs due to the less of micro- and small mesopores. The LiPSs formed on graphene surface easily dissolve in the bulk electrolytes, resulting in severe shuttle effect, and thus, lowering the capacity and Coulombic efficiency. Thus, the graphene surface must be modified to retain the LiPSs, which is the key to having a high rate performance coupling with a long cyclic stability. Anchoring metal oxides/chalcogenides, such as $\mathrm{TiO}_{2}[14$, 20], $\mathrm{MnO}_{2}$ [21] and $\mathrm{CoS}_{2}$ [22], onto graphene surface have been proved as a potential solution. These components have strong interaction with polar LiPSs which act as chemical adsorption sites to partially block the shuttling of LiPSs. In recent reports, the introduction of catalytic components, like $\mathrm{Pt}$ and $\mathrm{Ni}$, which not only show the adsorption ability towards the soluble LiPSs but also catalyse the conversion of insoluble LiPSs to long-chain LiPSs and sulfur during the charging process, greatly improves the capacity and reduces the overpotential of cathode [23, 24]. However, in many cases, these metal-based components require complicated preparation process and high cost and some oxides like $\mathrm{TiO}_{2}$ has high reactivity with electrolyte, leading to unexpected safety issue [25].

In this work, we introduce $\mathrm{Fe}_{2} \mathrm{O}_{3}$ nano-particles (NPs) onto graphene sheets and prepare a three-dimensional (3D) $\mathrm{Fe}_{2} \mathrm{O}_{3}$-graphene hybrid (denoted Fe-PGM) by a one-pot strategy. The $\mathrm{Fe}_{2} \mathrm{O}_{3}$ NPs can restrain shuttling of polysulfides by chemically adsorbing LiPSs thermodynamically as other oxides do, and more promisingly, accelerate the transformation of the soluble LiPSs to insoluble products, which have never been observed, as demonstrated by 
Figure 1. As a result, the utilization of sulfur is greatly improved and the shuttling of LiPSs is largely restrained. Moreover, the hybrid has a 3D hierarchical conductive graphene framework, which facilitates rapid ion/electron transfer while provides void space to accommodate sulfur and its volume expansion [26-29]. Therefore, the hybrid host holding sulfur (Fe-PGM-S) shows excellent rate capability with the capacity of 704.6 and $565.3 \mathrm{mAh} \mathrm{g}^{-1}$ at 2 and $5 \mathrm{C}$ respectively, and retains an excellent cyclic stability with an ultralow capacity fade rate of $0.049 \%$ per cycle at 5 C over 1000 cycles.

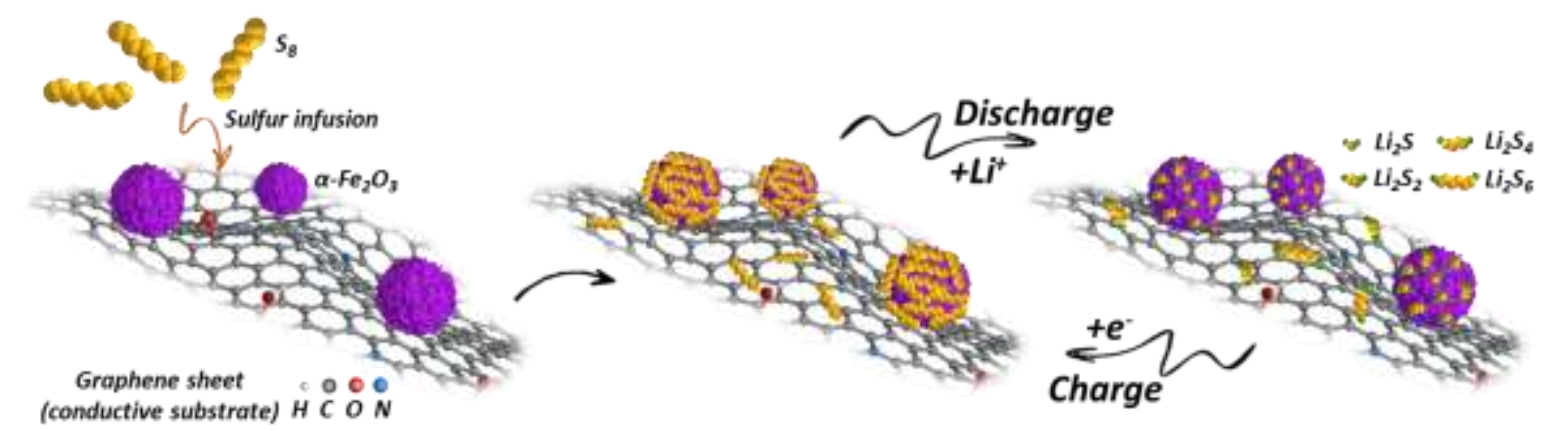

Figure 1. Schematic of the conversion process of sulfur on the graphene surface with $\mathrm{Fe}_{2} \mathrm{O}_{3} \mathrm{NPs}$.

\section{Experimental Section}

\subsection{Preparation of Fe-PGM}

Graphite oxide was synthesized from natural graphite by a modified Hummers method as reported previously [30, 31]. A graphene oxide (GO) colloidal solution $\left(2 \mathrm{mg} \mathrm{mL}^{-1}\right)$ was prepared by a sonication treatment for $2 \mathrm{~h}$ with graphite oxide $(160 \mathrm{mg})$ in deionized water $(80$ $\mathrm{mL}$ ). Typically, $3.2 \mathrm{mmol} \mathrm{FeCl}_{3}$ solution $(5 \mathrm{~mL})$ was slowly introduced into the abovementioned $80 \mathrm{~mL}$ GO colloidal solution. Then, it was stirred for $2 \mathrm{~h}$ to form uniform mixture. A moderate amount of aqueous ammonia (25 wt. \%) was added in these mixtures by dropwise until the $\mathrm{pH}$ value was up to 10 . Next, $1.3 \mathrm{~mL}$ hydrazine hydrate solution $\left(85 \mathrm{mg} \mathrm{mL}^{-1}\right)$ was dropped 
into the above mixtures at a speed of $100 \mu \mathrm{L}$ per 5 minute under stirring. The mixture was transferred into a $100 \mathrm{~mL}$ Teflon-lined stainless steel autoclave, followed by hydro-thermal treatment at $180{ }^{\circ} \mathrm{C}$ for $6 \mathrm{~h}$. The resulting product was obtained as a hydrogel. Later, it was washed with deionized water and then freeze-dried for $24 \mathrm{~h}$ to produce Fe-PGM. In the same process, the Fe-PGM-2 sample was obtained by reduce $3.2 \mathrm{mmol} \mathrm{FeCl}_{3}$ solution $(5 \mathrm{~mL})$ to 1.6 mmol.

\subsection{Preparation of PGM and $\alpha-\mathrm{Fe}_{2} \mathrm{O}_{3}$}

For comparison, the materials of PGM or $\alpha-\mathrm{Fe}_{2} \mathrm{O}_{3}$ were also prepared under the same conditions to prepare the Fe-PGM sample just without $\mathrm{FeCl}_{3}$ solution or $\mathrm{GO}$, respectively.

\subsection{Preparation of Fe-PGM-S, Fe-PGM-2-S and PGM-S}

The mixture of Fe-PGM (200 mg) and pure sulfur $(300 \mathrm{mg})$ was heat-treated at $155{ }^{\circ} \mathrm{C}$ for $12 \mathrm{~h}$ in Ar atmosphere to produce the Fe-PGM-S sample. For comparison, the samples of Fe-PGM-2$\mathrm{S}$ and PGM-S were also prepared under the same conditions using Fe-PGM-2 and PGM as the host materials, respectively.

\subsection{Electrode fabrication}

The cathode electrodes were fabricated by simply pressing the mixture of $80 \mathrm{wt}$. $\%$ active materials (as-synthesized Fe-PGM-S, Fe-PGM-2-S or PGM-S), 10 wt. \% conductive carbon black, and 10 wt. \% Polytetrafluoroethylene (PTFE) onto the nickel foam current collector. The mass loading of active materials was about $1 \mathrm{mg} \mathrm{cm}^{-2}$.

\subsection{Electrochemical Measurement}

A test cell was assembled with the above-mentioned cathode electrode and lithium metal anode electrode in a 2032 type stainless steel coin cell, where the electrolyte was $1.0 \mathrm{M}$ lithium bis-trifluoromethanesulphonylimide (LiTFSI) dissolved in 1, 3-dioxolane and 1, 2- 
dimethoxyethane (DOL: DME, 1:1 volume) ) with 0.5 wt. $\% \mathrm{LiNO}_{3}$ additive. The electrochemical performances were examined by cyclic voltammetry (CV) on CHI660E electrochemical workstation and galvanostatic charge/discharge using a LAND instrument. All the tests were carried out at room temperature (about $25^{\circ} \mathrm{C}$ ).

\subsection{Materials characterization}

The morphologies of all the samples were visualized by scanning electron microscopy (SEM, Hitachi S-4800, Japan) and high-resolution transmission electron microscopy (HR-TEM, F30 FEI). The nitrogen adsorption/desorption data was measured at $77 \mathrm{~K}$ using a 30 Micromeritics ASAP $2020 \mathrm{C}$ apparatus to determine the specific surface area and the pore size distribution. Raman spectra were measured by a multi-wavelength micro-Raman spectrometer (LabRAM HR800, HORIBA Jobin Yvon, Japan) using an Ar-ion laser of $532 \mathrm{~nm}$. The X-ray diffraction (XRD) measurements were conducted at room temperature using a Rigaku diffractometer (D/MAX-2500, Japan) equipped with $\mathrm{Cu}-\mathrm{K} \alpha$ radiation $(\lambda=0.154056 \mathrm{~nm})$. The fraction of $\alpha$ $\mathrm{Fe}_{2} \mathrm{O}_{3}$ in the Fe-PGM composite was calculated using thermogravimetric analysis (TG, Netzsch STA 449F3, Germany) in an air atmosphere at a heating rate of $5{ }^{\circ} \mathrm{C} / \mathrm{min}$ to $500{ }^{\circ} \mathrm{C}$, and the weight percentage contents of sulfur in the Fe-PGM-S, Fe-PGM-2-S, PGM-S and pure sulfur were measured in a $\mathrm{N}_{2}$ flow at the same condition. X-ray photoelectron spectroscopy (XPS ESCALAB 250Xi, US) analyses were conducted to analyze the surface species and chemical states of Fe-PGM-S and PGM-S.

\subsection{Density functional theory calculations}

Calculations were performed using spin-polarized density functional theory as implemented in Vienna Ab initio Simulation Package (VASP) [32] with the projected augmented wave (PAW) approach [33] and generalized gradient approximation by Perdew-Burke-Ernzerhof [34] with a 
Hubbard U correction [35]. An effective U value of $4.3 \mathrm{eV}$ [36] for Fe was chosen in our calculations. The plane-wave energy cutoff was set to be $500 \mathrm{eV}$. The vdw interactions were incorporated by the optB88 exchange functional [37]. A $10.1 \AA ̊$ × $10.1 \AA$ supercell of (0001) surface of $\alpha-\mathrm{Fe}_{2} \mathrm{O}_{3}$ with 12 atomic layers and a $\Gamma$-centered Monkhorst-Pack $5 \times 5 \times 1$ grids [38] in

k-point sampling were used. The vacuum distance larger than $10 \AA$ was applied in calculations to remove the interaction between successive slabs. In structural optimization, the lower six atomic layers of the slabs were fixed while allowing other atoms to fully relax until the force was converged to $0.01 \mathrm{eV} / \AA ̊$.

\section{Results and Discussion}

The integrated macrostructures of PGM, Fe-PGM and Fe-PGM-2 are shown in Figure S1a. The $\alpha-\mathrm{Fe}_{2} \mathrm{O}_{3}$ contents in the Fe-PGM and Fe-PGM-2 are 11.3 wt. $\%$ and 24.5 wt. $\%$ measured by thermal gravimetric analysis (TGA) (Figure S1b), and the PGM is free of $\mathrm{Fe}_{2} \mathrm{O}_{3}$ for comparison. The hybrids containing sulfur, denoted PGM-S, Fe-PGM-S and Fe-PGM-2-S (Figure S2), were prepared with above samples by a melt-diffusion method in Ar atmosphere, and the sulfur content was about $60 \mathrm{wt} . \%$ in these hybrids.

Figure 2 shows the structures of Fe-PGM and Fe-PGM-S that are characterized by scanning electron microscopy (SEM) and transmission electron microscopy (TEM). The PGM shows a 3D hierarchical porous structure with many large pores (Figure S1c and Figure S1f). At the same time, the Fe-PGM also apparently shows similar structure with an interconnected framework but fewer large macropores (Figure 2a and Figure S1d). It is noted that the $\mathrm{Fe}^{3+}$ ions introduced in the preparation process may promote the assembly of GO, and may also act as linkers between GO sheets to help form more mesopores indicated by the large hysteresis loops in $\mathrm{N}_{2}$ adsorptiondesorption isotherms (Figure S3) [39, 40]. This porous structure can accommodate the volume 
expansion of sulfur and ensure the fast ion diffusion, while the interconnected graphene sheets form 3D conductive network, ensuring fast electron transfer. The high-magnification SEM images in Figure 2b and Figure S1g show the uniform distribution of $\alpha-\mathrm{Fe}_{2} \mathrm{O}_{3} \mathrm{NPs}$ on the graphene surface, and the size of these NPs is about $20 \mathrm{~nm}$ as shown in Figure 2c. The high resolution TEM (HRTEM) image in Figure 2d demonstrates that the fringe spacing is $0.25 \mathrm{~nm}$, corresponding to the interplanar spacing of (110) facet of single-crystalline trigonal $\alpha-\mathrm{Fe}_{2} \mathrm{O}_{3}$. This is in consist with the selected area electron diffraction (SAED) pattern (inset of Figure 2d). The diffraction peaks of the XRD pattern in Figure $2 \mathbf{l}$ are also well agreed with the $\alpha-\mathrm{Fe}_{2} \mathrm{O}_{3}$ (JCPDS 33-0664) [41]. Compared with Fe-PGM (Figure 2c), the TEM image of Fe-PGM-S hybrid (Figure 2e) shows similar morphology to that of Fe-PGM, but the elemental mapping shows the uniformly infiltrated sulfur on the graphene surface (Figure $2 \mathbf{f}$ and $\mathbf{2 k}$ ). In addition, Figure $2 \mathbf{h}$ reveals $\mathrm{N}$-doping due to the introduced $\mathrm{NH}_{3} \cdot \mathrm{H}_{2} \mathrm{O}$ and hydrazine hydrate in the hydrothermal process, which will be beneficial to improve the conductivity and enhance the interaction between graphene surface and LiPSs according to previous reports [2, 42]. The specific surface area (SSA) of Fe-PGM calculated by Brunauer-Emmett-Teller (BET) method reduces from about 300 to $7 \mathrm{~m}^{2} \mathrm{~g}^{-1}$ for Fe-PGM-S, suggesting the sulfur has been infiltrated in the hierarchical porous structure. The pore size analysis (Figure S3, Table S1) further shows that the micropores and small mesopores in the Fe-PGM-S hybrid disappear, but large mesopores and macropores still exist, leaving abundant ion transport channels to ensure good rate capability. In addition, both the XRD (Figure 2I) and Raman (Figure S4) results also show the incorporation of sulfur for Fe-PGM-S hybrid. The typical diffraction peak of GO located at $12^{\circ}$ disappears and shifts to a broad and weak peak at around $25^{\circ}$ for PGM, confirming that during the hydrothermal 
assembly process, the restacking of reduced GO is avoided due to the formation of 3D porous structure.
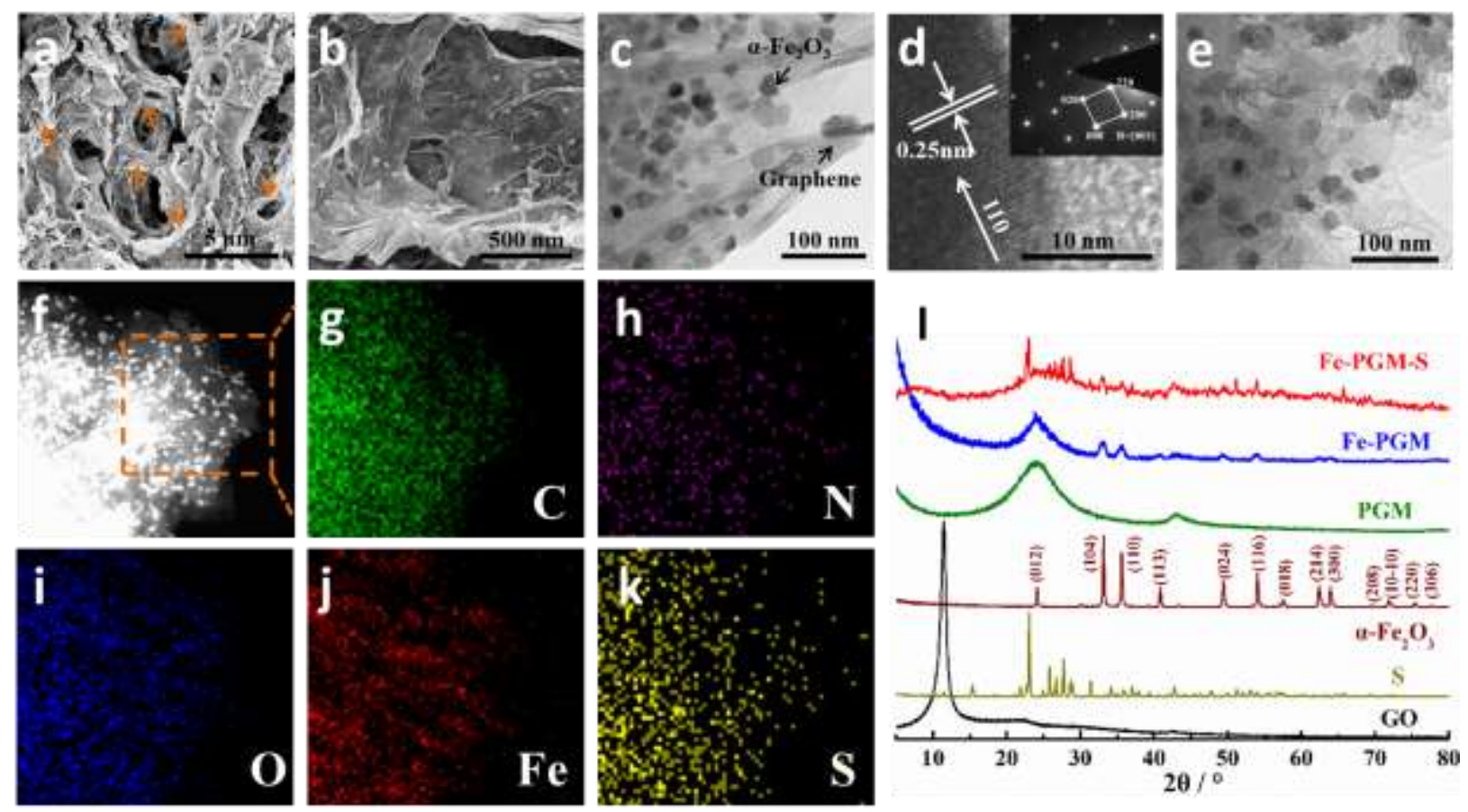

Figure 2. Morphology demonstration of the Fe-PGM and Fe-PGM-S hybrids. SEM (a, b)

and TEM (c) images of Fe-PGM; (d) HRTEM images of the $\alpha-\mathrm{Fe}_{2} \mathrm{O}_{3}$ nano-particles in Fe-PGM with a SAED pattern of the $\alpha-\mathrm{Fe}_{2} \mathrm{O}_{3}$ as the Inset; TEM image (e), STEM image (f), element mappings (g-k) of Fe-PGM-S; (l) XRD patterns of GO, sulfur, $\alpha-\mathrm{Fe}_{2} \mathrm{O}_{3}$, PGM, Fe-PGM and FePGM-S.

In order to confirm the chemical composition of the Fe-PGM-S hybrid and the interaction between $\alpha-\mathrm{Fe}_{2} \mathrm{O}_{3}$ and LiPSs, X-ray photoelectron spectroscopy (XPS) measurements were carried out. In the fitted C1s profile shown in Figure 3a, there are four peaks at 284.6, 285.8, 287.3 and $289.1 \mathrm{eV}$, corresponding to the bonds of $\mathrm{C}-\mathrm{C} / \mathrm{C}=\mathrm{C}, \mathrm{C}-\mathrm{O} / \mathrm{C}-\mathrm{N} / \mathrm{C}-\mathrm{S}, \mathrm{C}=\mathrm{O}$ and $\mathrm{O}-\mathrm{C}=\mathrm{O}$, respectively.43, 44 Compared with the $\mathrm{C} 1$ s profile of $\mathrm{GO}$ (Figure $\mathbf{S 5}$ ), the $\mathrm{C}=\mathrm{C}$ bond of Fe-PGM (located at $284.6 \mathrm{eV}$ ) becomes much stronger and narrower, suggesting the successful reduction 
of GO. The $\mathbf{N} 1$ s spectrum in Figure $3 \mathbf{b}$ can be resolved into two peaks with the binding energies of 398.9 and $400.3 \mathrm{eV}$, corresponding to the pyridinic $\mathrm{N}$ and pyrrolic $\mathrm{N}[45,46]$.These doped nitrogen atoms increase the polarity of graphene surface, which can help improve the adsorption of LiPSs onto graphene [47, 48]. In the Fe 2p spectrum (Figure 3c and Figure S6), the peaks located at 711.1 and $724.5 \mathrm{eV}$ are ascribed to the $\mathrm{Fe} 2 \mathrm{p}_{3 / 2}$ and $\mathrm{Fe} 2 \mathrm{p}_{1 / 2}$ of $\alpha-\mathrm{Fe}_{2} \mathrm{O}_{3}$ [49]. It is noted that, for the Fe-PGM-S hybrid, the other two peaks located at 708.8 and $722.2 \mathrm{eV}$ are ascribed to $\mathrm{Fe}-\mathrm{S}$ bond $[50,51]$, indicating the strong interaction of sulfur-based species with $\alpha-\mathrm{Fe}_{2} \mathrm{O}_{3}$. This interaction is further proved by the peak at $162.6 \mathrm{eV}$ in the $\mathrm{S} 2 \mathrm{p}$ spectrum (Figure 3d) [50, 51], which is not observed in the PGM-S hybrid (Figure 3e), further confirming the interaction between $\alpha-\mathrm{Fe}_{2} \mathrm{O}_{3}$ and sulfur. In order to further prove the interaction of $\alpha-\mathrm{Fe}_{2} \mathrm{O}_{3}$ with LiPSs, an equivalent mass of the PGM and Fe-PGM were separately added to a solution of $\mathrm{Li}_{2} \mathrm{~S}_{6}$ dissolved in 1,2-dimethoxyethane (DME) with exactly the same amount and concentration. After 30 minutes, the solution containing the Fe-PGM shows completely colourless, whereas the solution with PGM still keeps light yellow as shown in Figure 3f. 

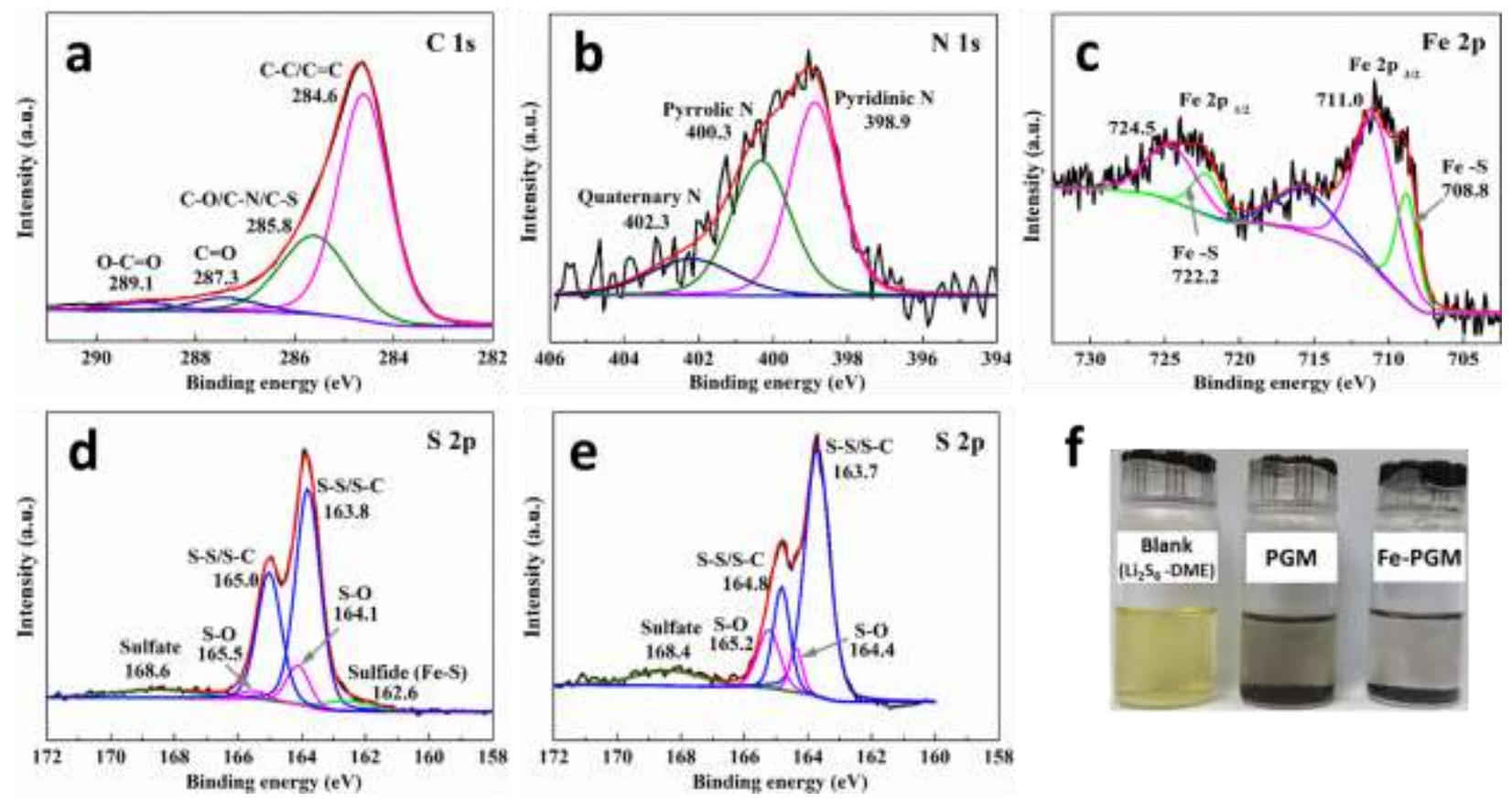

Figure 3. Demonstration of the strong interaction of LiPSs with $\boldsymbol{\alpha}_{-}-\mathrm{Fe}_{2} \mathrm{O}_{3} \cdot \mathrm{C}$ 1s (a), N 1s (b),

Fe $2 p$ (c) and S 2p (d) XPS curves of Fe-PGM-S; (e) S 2p XPS curve of the PGM-S hybrid; (f) Optical photos of PGM and Fe-PGM materials soaked in $\mathrm{Li}_{2} \mathrm{~S}_{6}$-DME solution.

To show whether the $\alpha-\mathrm{Fe}_{2} \mathrm{O}_{3}$ NPs are beneficial to the electrochemical performance, detailed electrochemical measurements were carried out using coin cells (2032) with Li metal as the anode. The cyclic voltammetry (CV) profiles of PGM-S, Fe-PGM-S and Fe-PGM-2-S (Figure 4 a-c and Figure S7) were obtained between 1.7 and $2.8 \mathrm{~V}$ at a scan rate of $0.1 \mathrm{mV} \mathrm{s}^{-1}$. In the cathodic reduction process, there are two characteristic reductive peaks at approximately 2.31 (vs. $\mathrm{Li}^{+} / \mathrm{Li}$, denoted $\left.\mathrm{E} 1_{\mathrm{pc}}\right)$ and $2.05 \mathrm{~V}$ (denoted $\left.\mathrm{E} 2_{\mathrm{pc}}\right)$ in the 2nd cycle. $\mathrm{E} 1_{\mathrm{pc}}$ is related to long chain LiPSs $\left(\operatorname{Li}_{2} \mathrm{~S}_{\mathrm{n}}, 4 \leq \mathrm{n} \leq 8\right)$ from sulfur and $\mathrm{E} 2_{\mathrm{pc}}$ is derived from the reduction of long-chain LiPSs to short-chain LiPSs such as $\mathrm{Li}_{2} \mathrm{~S}_{2}$ and $\mathrm{Li}_{2} \mathrm{~S}$ represented as the reaction (1) [52-54], corresponding to the typical two-discharge plateaus in the galvanostatic discharge curve in Figure 4e. Note that with the increase of $\alpha-\mathrm{Fe}_{2} \mathrm{O}_{3}$ content, the $\mathrm{E} 2_{\mathrm{pc}}$ shows higher intensity and narrower peak, suggesting the reaction occurred at this potential (Reaction (1)) is greatly enhanced. This means 
the transformation of soluble LiPSs to final insoluble products is promoted, which substantially decreases the possibilities for the LiPSs shuttling into the electrolyte. This is different from the catalytic behaviour of $\mathrm{Pt}$ or $\mathrm{Ni}$ that enhances the conversion of insoluble LiPSs to long-chain LiPSs [23, 24].

$$
L i_{2} S_{4}+L i^{+}+e^{-} \rightarrow L i_{2} S_{2}, L i_{2} S
$$

During the oxidation process, the peak at 2.35 (denoted $E 1_{\mathrm{pa}}$ ) is related to the formation of $\mathrm{Li}_{2} \mathrm{~S}_{\mathrm{n}}(\mathrm{n}>2)$, and this reaction continues until LiPSs are completely transformed into elemental sulfur at $2.39 \mathrm{~V}$ (denoted $\mathrm{E} 2 \mathrm{pa}$ ) (Reaction (2)) [55]. Note that with the increase of $\alpha-\mathrm{Fe}_{2} \mathrm{O}_{3}$ content, $\mathrm{E} 2_{\mathrm{pa}}$ shifts to a lower potential and eventually merges with $\mathrm{E} 1_{\mathrm{pa}}$, meaning the activation energy for Reaction (2) is largely decreased which promotes the reaction from soluble LiPSs to sulfur. This also reduces the possibility for the shuttling of LiPSs. In the first cycle, a more obvious reaction potential decrease appears (from 2.42 V for PGM-S to $~ 2.40 \mathrm{~V}$ for Fe-PGM-S and Fe-PGM-2-S) (Figure S8). These results clearly suggest the $\mathrm{Fe}_{2} \mathrm{O}_{3}$ NPs, as expected, help trap the LiPSs, and more promisingly promote the transformation of soluble intermediate products to insoluble products as electrocatalysts used in Li-S batteries [56, 57]. Thus, the shuttling of LiPSs is greatly suppressed. Besides, the 3rd and 4th CV curves of the Fe-PGM-S electrode are almost coincided with each other, indicating the high stability of these hybrids.

$$
L i_{2} S_{n}-L i^{+}-e^{-} \rightarrow L i_{2} S_{n-1}+S_{8} \quad(n>2)
$$

In Figure 4d-f, the typical two discharge plateaus for the three cathodes can be well observed even at a very high rate of $5 \mathrm{C}$, which suggests fast reaction kinetic due to the hierarchical porous structure and interconnected conductive network. It is noted that the Fe-PGM-S cathode show the lowest overpotential $(\Delta \mathrm{E}=177 \mathrm{mV})$ that is calculated by the voltage difference between the 
first charge plateau and the second discharge plateau in the first cycle, compared to the $\Delta \mathrm{E}$ value of $201 \mathrm{mV}$ for the PGM-S and $190 \mathrm{mV}$ for the Fe-PGM-2-S. This indicates the introduction of $\mathrm{Fe}_{2} \mathrm{O}_{3}$ NPs enhances the redox reaction kinetics. However, too many $\alpha-\mathrm{Fe}_{2} \mathrm{O}_{3}$ NPs lower the conductivity of the electrode, and thus, the Fe-PGM-2-S cathode shows slightly larger $\Delta \mathrm{E}$ value than Fe-PGM-S. Moreover, Tafel plots (Figure 4g) and corresponding exchange current density values (Table S2) have been calculated from potentiostatic polarization experiments to understand the effect of $\alpha-\mathrm{Fe}_{2} \mathrm{O}_{3}$ on the redox reaction kinetics. The Fe-PGM-S electrode shows the largest exchange current densities for both the cathodic process and the anodic process (denoted $\mathrm{I}_{0, \mathrm{c}}, \mathrm{I}_{0, \mathrm{a}}$ respectively), which are 3.58 and $7.03 \mathrm{~mA} \mathrm{~cm}^{-2}$, compared to the PGM-S (2.28 and $4.81 \mathrm{~mA} \mathrm{~cm}^{-2}$ ) and Fe-PGM-2-S (3.46 and $\left.4.96 \mathrm{~mA} \mathrm{~cm}^{-2}\right)$. The increase in exchange current density clearly validates the enhancement of the conversion rate of intermediate, soluble LiPSs into insoluble products in both charge and discharge processes [58].

The fast-redox reaction kinetics induces the highest discharge capacity of $1571.2 \mathrm{mAh} \mathrm{g}^{-1}$ for the Fe-PGM-S at $0.3 \mathrm{C}$. With the increase of the current density, Fe-PGM-S cathode shows the discharge capacities of $1001.5,829.9,704.6$ and $565.3 \mathrm{mAh} \mathrm{g}^{-1}$ at $0.5,1,2$, and $5 \mathrm{C}$, respectively. An excellent high-rate cycling performance of the Fe-PGM-S hybrid is presented in Figure 5. As shown in Figure 5a, the Fe-PGM-S keeps the highest capacity retention (54\%) and a lower fading rate $(0.09 \%$ per cycle) with nearly $100 \%$ Coulombic efficiency over 500 cycles compared with Fe-PGM-2-S (34.0\%, 0.13\% per cycle) and PGM-S (31.4\%, 0.14\% per cycle) at a current rate of $2 \mathrm{C}$. After 1000 cycles at a higher rate of $5 \mathrm{C}$ (Figure 5b), the Fe-PGM-S electrode continues to maintain its superiority on the cycling performance with the capacity decay of only $0.049 \%$ per cycle, while the capacities of Fe-PGM-2-S and PGM-S electrodes fade rapidly over 1000 cycles $(0.064 \%$ and $0.080 \%$ per cycle, respectively). This excellent 
performance for Li-S batteries based on the Fe-PGM composite should be ascribed to the following aspects. First, the 3D hierarchical graphene-based framework not only provides the fast ion and electron transfer channels, but also retains the shuttling of LiPSs by physical adsorption to some extent. Then, $\mathrm{N}$-doping onto graphene and the anchored $\mathrm{Fe}_{2} \mathrm{O}_{3}$ further increases the adsorption towards the LiPSs by chemical bonding. Last but the most importantly, the $\alpha-\mathrm{Fe}_{2} \mathrm{O}_{3}$ nanoparticles promotes the transformation of the soluble LiPSs to insoluble products in both charging and discharging processes, greatly suppressing the shuttling of LiPSs. 

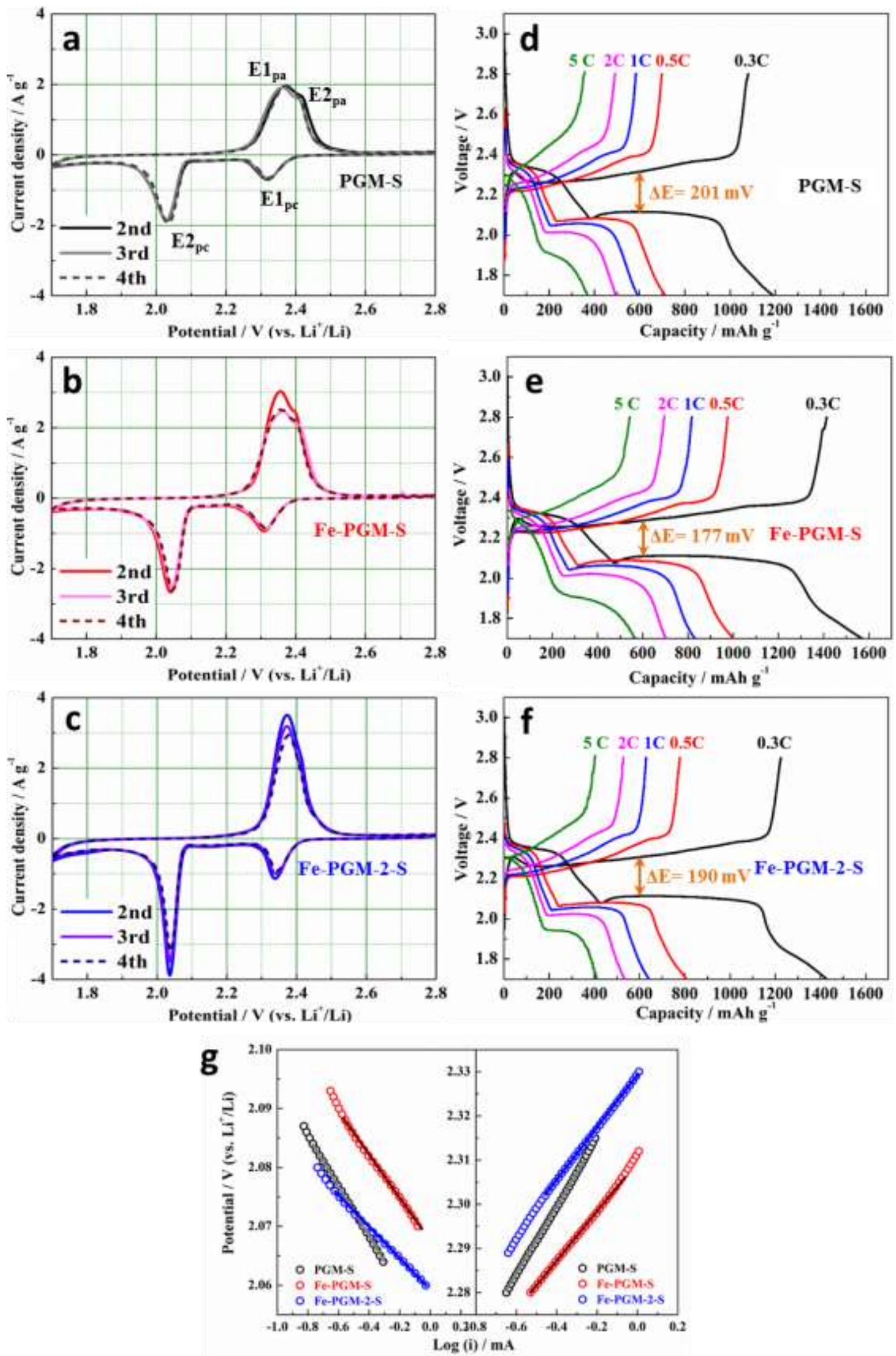
Figure 4. Electrochemical performance of the Fe-PGM-S hybrid as a cathode material for Li-S batteries in comparison to those of the PGM-S and Fe-PGM-2-S hybrids. CV profiles of PGM-S (a), Fe-PGM-S (b) and Fe-PGM-2-S (c) at scan rate of $0.1 \mathrm{mV} \mathrm{s}^{-1}$; the charge/discharge profiles of PGM-S (d), Fe-PGM-S (e) and Fe-PGM-2-S (f) at various rates ranging from $0.3 \mathrm{C}$ to $5 \mathrm{C} ; \mathrm{g}$ ) Tafel plots for corresponding reduction and oxidation reactions.
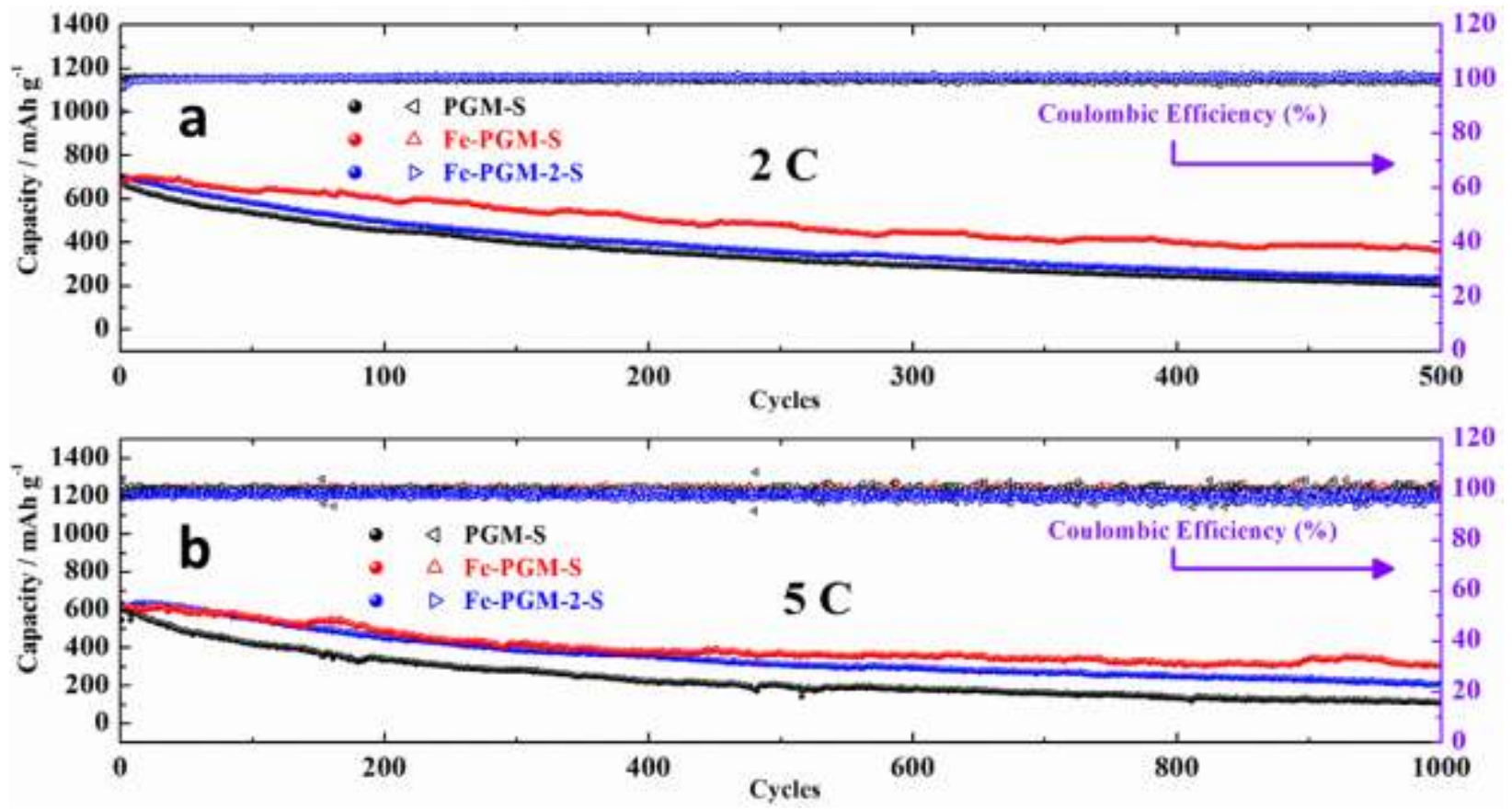

Figure 5. Cycling performance. The Fe-PGM-S hybrid as a cathode material for $\mathrm{Li}-\mathrm{S}$ batteries in comparison to those of the PGM-S and Fe-PGM-2-S hybrids, respectively at $2 \mathrm{C}$ (a) and 5 $\mathrm{C}(\mathrm{b})$. The Coulombic efficiency is shown on the right y-axis.

To further understand the origin of electrochemical performance improvement observed in Li$\mathrm{S}$ batteries based on incorporation of $\alpha-\mathrm{Fe}_{2} \mathrm{O}_{3}$ into carbon materials, we investigate the interaction between LiPSs (including $\mathrm{S}_{8}$ ) and $\alpha-\mathrm{Fe}_{2} \mathrm{O}_{3}$ by density functional theory calculations. The Fe-terminated (0001) surface of $\alpha-\mathrm{Fe}_{2} \mathrm{O}_{3}$ is chosen, as it is the most stable surface structure under the low oxygen pressure condition, to study the adsorption behavior of S-containing 
clusters $\left(\mathrm{S}_{8}, \mathrm{Li}_{2} \mathrm{~S}\right.$ and $\left.\mathrm{Li}_{2} \mathrm{~S}_{2 \mathrm{n}}, \mathrm{n}=1,2,3,4\right)$. The adsorption energies $\left(E_{a \mathrm{ds}}\right)$ are defined by:

$$
E_{a \mathrm{ds}}=E_{\mathrm{Fe}_{2} \mathrm{O}_{3}}+E_{S}-E_{\text {total }}
$$

where $E_{\mathrm{Fe}_{2} \mathrm{O}_{3}}, E_{S}$, and $E_{\text {total }}$ are the total energies of the (0001) surface of $\alpha-\mathrm{Fe}_{2} \mathrm{O}_{3}$, the isolated S-containing cluster and the surface of $\alpha-\mathrm{Fe}_{2} \mathrm{O}_{3}$ bonded with different S-containing clusters, respectively. As shown in Table 1, the calculated adsorption energies of S-containing clusters on the (0001) surface of $\alpha-\mathrm{Fe}_{2} \mathrm{O}_{3}$ increase from $2.04 \mathrm{eV}$ for $\mathrm{S}_{8}$ to $4.85 \mathrm{eV}$ for $\mathrm{Li}_{2} \mathrm{~S}$. This strong binding is important for not only loading sulfur, resulting in improving the capacity, but anchoring LiPSs and preventing them from dissolving into the electrolyte, thus inhibiting the capacity loss. For comparison, we also calculated the $E_{a \mathrm{ds}}$ of S-containing clusters adsorption on graphene with non-polar characteristics. The calculated $E_{a \mathrm{ds}}$ increases from $0.65 \mathrm{eV}$ for $\mathrm{Li}_{2} \mathrm{~S}$ to 1.10 for $\mathrm{Li}_{2} \mathrm{~S}_{8}$, then decreases to $0.89 \mathrm{eV}$ for $\mathrm{S} 8$, as listed in Table 1 . It is clearly seen that the adsorption energies $E_{a \text { ds }}$ increase with increased cluster size of S-containing clusters. The large different $E_{a \text { ds }}$ of S-containing clusters adsorption on $\alpha-\mathrm{Fe}_{2} \mathrm{O}_{3}$ and graphene is due to the different types of binding mechanisms. The interaction between $\mathrm{S}_{8}$ and $\alpha-\mathrm{Fe}_{2} \mathrm{O}_{3}$ are mainly due to the weak S-Fe bonds. While for LiPSs, as shown in Figure 6, the computed differential charge density of $\mathrm{Li}_{2} \mathrm{~S}_{4}$ adsorption on $\alpha-\mathrm{Fe}_{2} \mathrm{O}_{3}$ clearly shows the strong bonding between $\mathrm{Li}-\mathrm{O}$ and $\mathrm{Fe}-\mathrm{S}$ ions, which is quite different from the dipole-dipole interaction between the $\mathrm{Li}_{2} \mathrm{~S}_{4}$ and graphene. So we can conclude that the interaction between S-containing clusters and graphene mainly comes from the vdw interaction. For all S-containing clusters on the surface of $\alpha-\mathrm{Fe}_{2} \mathrm{O}_{3}$, the $E_{a \mathrm{ds}}$ increases when the sulfur chains become shorten. This suggests that the Li-O and Fe-S bonds become stronger, which is closely correlated to the charge transferred from S-containing clusters to the substrate of $\alpha-\mathrm{Fe}_{2} \mathrm{O}_{3}$. As we can see in Table 1, the binding becomes strengthened from $2.04 \mathrm{eV}$ for $\mathrm{S}_{8}$ to $4.85 \mathrm{eV}$ for $\mathrm{Li}_{2} \mathrm{~S}$, electrons transferred increases from 0.11 e to $0.82 \mathrm{e}$, 
respectively. The results clearly indicate that $\alpha-\mathrm{Fe}_{2} \mathrm{O}_{3}$ largely enhance the interaction of the sulfur host and LiPSs, and the ongoing calculations are expected to show the fast transformation of the adsorbed LiPSs into insoluble sulfur species. All these indicate the key role of $\alpha-\mathrm{Fe}_{2} \mathrm{O}_{3}$ in restraining the shuttering of the soluble LiPSs, improving both the rate and cycling performances.

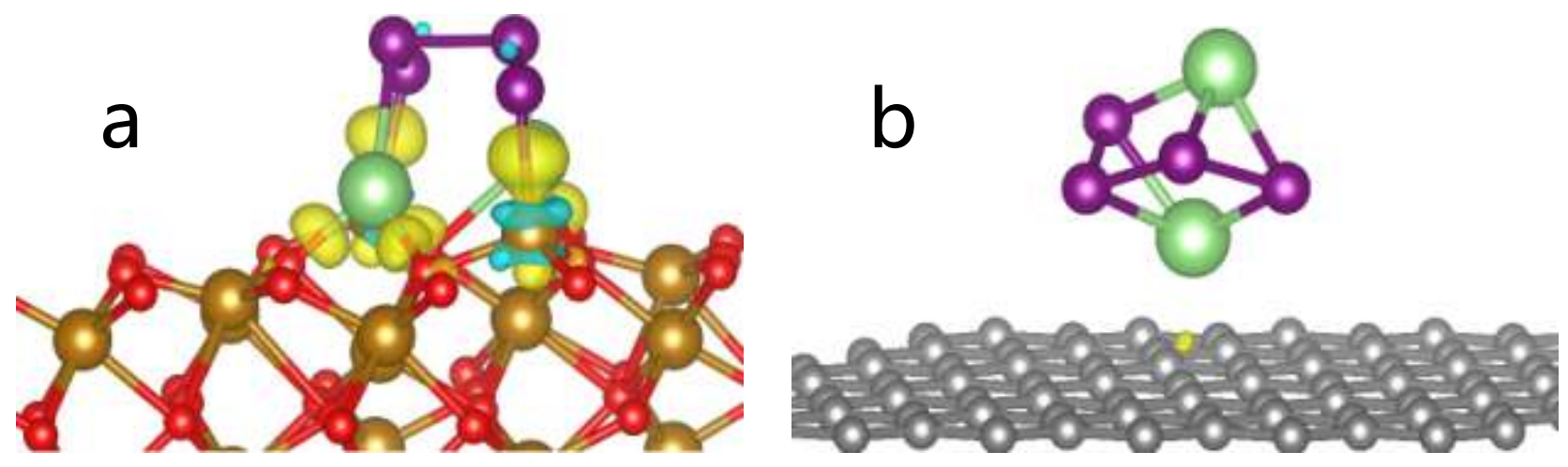

Figure 6. Computed differential charge density of the $\mathrm{Li}_{2} \mathrm{~S}_{4}$ adsorption on (a) $\alpha-\mathrm{Fe}_{2} \mathrm{O}_{3}$ and (b) graphene. The differential charge densities are plotted with isosurface value of $0.007 \mathrm{e} / \AA^{3}$, where the yellow and blue represent the electron-rich and electron-deficient regions, respectively. The green, purple, yellow, red and grey balls represent the $\mathrm{Li}, \mathrm{S}, \mathrm{Fe}, \mathrm{O}$ and $\mathrm{C}$ atoms, respectively. Table 1. Adsorption energies $\left(E_{a \mathrm{ds}}\right)$ of S-containing clusters adsorption on graphene and $\alpha$ $\mathrm{Fe}_{2} \mathrm{O}_{3}$. For LiPSs, the charge transferred to $\alpha-\mathrm{Fe}_{2} \mathrm{O}_{3}$ are also listed.

\begin{tabular}{cccc}
\hline & Graphene & \multicolumn{2}{c}{$\alpha-\mathrm{Fe}_{2} \mathrm{O}_{3}$} \\
\cline { 2 - 4 } & $\mathrm{E}_{\text {ads }}(\mathrm{eV})$ & $\mathrm{E}_{\text {ads }}(\mathrm{eV})$ & charge transfer \\
\hline $\mathrm{S}_{8}$ & 0.89 & 2.04 & 0.11 \\
$\mathrm{Li}_{2} \mathrm{~S}_{8}$ & 1.10 & 3.78 & 0.57 \\
$\mathrm{Li}_{2} \mathrm{~S}_{6}$ & 0.93 & 4.11 & 0.68 \\
$\mathrm{Li}_{2} \mathrm{~S}_{4}$ & 0.72 & 4.09 & 0.64 \\
$\mathrm{Li}_{2} \mathrm{~S}_{2}$ & 0.74 & 4.20 & 0.59 \\
$\mathrm{Li}_{2} \mathrm{~S}$ & 0.65 & 4.85 & 0.82 \\
\hline
\end{tabular}




\section{Conclusions}

A Fe-PGM hybrid with a uniformly distributed $\alpha-\mathrm{Fe}_{2} \mathrm{O}_{3} \mathrm{NPs}$ on a $3 \mathrm{D}$ hierarchical porous graphene is prepared by a one-step hydrothermal treatment. The hybrid is used as a novel host for sulfur to realize the high rate and cyclic stability for the cathode of Li-S batteries. It is found that $\alpha-\mathrm{Fe}_{2} \mathrm{O}_{3}$ NPs not only build strong interaction with sulfur and polysulfide species, but also promote the transformation of the soluble polysulfides to insoluble products in both charge and discharge process, effectively suppressing the shuttling of the polysulfides and improving the sulfur utilization. Thus, the Fe-PGM-S hybrid shows high specific capacity, high-rate capability

and long cyclic stability, typically a high capacity of $565 \mathrm{mAh} \mathrm{g}^{-1}$ with an ultralow capacity fading of $0.049 \%$ per cycle over 1000 cycles at $5 \mathrm{C}$. This study clear shows the introduction of metal oxides in the cathode for Li-S battery may play a role beyond the chemical adsorption towards the polysulfides, and moreover, it indicates a strategy to design the high rate and long cycle life sulfur-based cathode by fastening the transformation of soluble polysulfides, which is important for developing practically useable Li-S batteries.

\section{Acknowledgments}

This work was supported by the National Basic Research Program of China (2014CB932400), National Natural Science Foundation of China (Nos. 51525204, 51502150 and 51232005), the Shenzhen Basic Research Project (JCYJ20150529164918734 and KQCX20140521161756227) and National Science Foundation for Post-doctoral Scientists of China (No. 2015M571038).

\section{References}

[1] A. Manthiram, S. H. Chung, C. Zu, Adv. Mater. 27 (2015) 1980-2006.

[2] G. Zhou, E. Paek, G. S. Hwang, A. Manthiram, Nat. Commun. 6 (2015) 7760. 
[3] Z. Liang, G. Zheng, W. Li, Z. W. Seh, H. Yao, K. Yan, D. Kong, Y. Cui, ACS Nano 8 (2014) $5249-5256$.

[4] J. Sun, Y. Sun, M. Pasta, G. Zhou, Y. Li, W. Liu, F. Xiong, Y. Cui, Adv. Mater. 28 (2016) 9797-9803.

[5] X.-B. Cheng, C. Yan, J.-Q. Huang, P. Li, L. Zhu, L. Zhao, Y. Zhang, W. Zhu, S.-T. Yang, Q. Zhang, Energy Storage Mater. 6 (2017) 18-25.

[6] F. Lu, M. Zhou, W. Li, Q. Weng, C. Li, Y. Xue, X. Jiang, X. Zeng, Y. Bando, D. Golberg, Nano Energy 26 (2016) 313-323.

[7] Q. Pang, X. Liang, C. Y. Kwok, J. Kulisch, L. F. Nazar, Adv. Energy Mater. (2016) 1601630.

[8] Z. Li, H. B. Wu, X. W. Lou, Energy Environ. Sci. 9 (2016) 3061-3070.

[9] A. Manthiram, Y. Fu, S.-H. Chung, C. Zu, Y.-S. Su, Chem. Rev. 114 (2014) 11751-11787.

[10] J. M. Tarascon, M. Armand, Nature 414 (2001) 359-367.

[11] Y. Yang, G. Zheng, Y. Cui, Chem. Soc. Rev. 42 (2013) 3018-3032.

[12] C. Barchasz, J.-C. Leprêtre, F. Alloin, S. Patoux, J. Power Sources 199 (2012) 322-330.

[13] Y. V. Mikhaylik, J. R. Akridge, J. Electrochem. Soc. 151 (2004) A1969-A1976.

[14] Z. W. Seh, W. Li, J. J. Cha, G. Zheng, Y. Yang, M. T. McDowell, P. C. Hsu, Y. Cui, Nat. Commun. 4 (2013) 1331.

[15] D.-W. Wang, Q. Zeng, G. Zhou, L. Yin, F. Li, H.-M. Cheng, I. R. Gentle, G. Q. M. Lu, J. Mater. Chem. A 1 (2013) 9382-9394.

[16] Q. Zhang, X. Cheng, J. Huang, H. Peng, F. Wei, New Carbon Mater. 29 (2014) 241-264.

[17] W. Lv, Z. Li, Y. Deng, Q.-H. Yang, F. Kang, Energy Storage Mater. 2 (2016) 107-138.

[18] C. Zhang, W. Lv, W. Zhang, X. Zheng, M.-B. Wu, W. Wei, Y. Tao, Z. Li, Q.-H. Yang, Adv. Energy Mater. 4 (2014) 1301565. 
[19] J. Liang, Z.-H. Sun, F. Li, H.-M. Cheng, Energy Storage Mater. 2 (2016) 76-106.

[20] J.-Y. Hwang, H. M. Kim, S.-K. Lee, J.-H. Lee, A. Abouimrane, M. A. Khaleel, I. Belharouak, A. Manthiram, Y.-K. Sun, Adv. Energy Mater. 6 (2016) 1501480.

[21] X. Liang, C. Hart, Q. Pang, A. Garsuch, T. Weiss, L. F. Nazar, Nat. Commun. 6 (2015) 5268.

[22] Z. Yuan, H.-J. Peng, T.-Z. Hou, J.-Q. Huang, C.-M. Chen, D.-W. Wang, X.-B. Cheng, F. Wei, Q. Zhang, Nano Lett. 16 (2016) 519-527.

[23] H. Al Salem, G. Babu, C. V. Rao, L. M. Arava, J. Am. Chem. Soc. 137 (2015) 11542-11545.

[24] G. Babu, K. Ababtain, K. Y. Ng, L. M. Arava, Sci. Rep. 5 (2015) 8763.

[25] Y.-B. He, M. Liu, Z.-L. Xu, B. Zhang, B. Li, F. Kang, J.-K. Kim, Energy Technol. 1 (2013) 668-674.

[26] Z. Chen, W. Ren, L. Gao, B. Liu, S. Pei, H. M. Cheng, Nat. Mater. 10 (2011) 424-428.

[27] G. Zhou, L.-C. Yin, D.-W. Wang, L. Li, S. Pei, I. R. Gentle, F. Li, H.-M. Cheng, ACS Nano 7 (2013) 5367-5375.

[28] Y. Xu, Z. Lin, X. Zhong, X. Huang, N. O. Weiss, Y. Huang, X. Duan, Nat. Commun. 5 (2014) 4554.

[29] Q. Zhang, Z. Xu, B. Lu, Energy Storage Mater. 4 (2016) 84-91.

[30] W. Lv, D.-M. Tang, Y.-B. He, C.-H You, Z.-Q. Shi, X.-C. Chen, C.-M. Chen, P.-X. Hou, C. Liu, Q.-H. Yang, ACS Nano 3 (2009) 3730-3736.

[31] C. Zhang, W. Lv, X. Xie, D. Tang, C. Liu, Q.-H. Yang, Carbon 62 (2013) 11-24.

[32] G. Kresse, J. Furthmüller, Comp. Mater. Sci. 6 (1996) 15-50.

[33] G. Kresse, D. Joubert, Phys. Rev. B 59 (1999) 1758-1775.

[34] J. P. Perdew, K. Burke, M. Ernzerhof, Phys. Rev. Lett. 77 (1996) 3865-3868. 
[35] S. L. Dudarev, G. A. Botton, S. Y. Savrasov, C. J. Humphreys, A. P. Sutton, Phys. Rev. B 57 (1998) 1505-1509.

[36] N. J. Mosey, P. Liao, E. A. Carter, J. Chem. Phys. 129 (2008) 014103.

[37] J. Klimeš, D. R. Bowler, A. Michaelides, Phys. Rev. B 83 (2011) 195131.

[38] H. J. Monkhorst, J. D. Pack, Phys. Rev. B 13 (1976) 5188-5192.

[39] H. Yin, S. Zhao, J. Wan, H. Tang, L. Chang, L. He, H. Zhao, Y. Gao, Z. Tang, Adv. Mater. 25 (2013) 6270-6276.

[40] W. Lv, C. Zhang, Z. Li, Q. H. Yang, J. Phys. Chem. Lett. 6 (2015) 658-668.

[41] Q. Pan, K. Huang, S. Ni, F. Yang, S. Lin, D. He, J. Phys. D: Appl. Phys. 42 (2009) 015417.

[42] S. Niu, W. Lv, C. Zhang, F. Li, L. Tang, Y. He, B. Li, Q.-H. Yang, F. Kang, J. Mater. Chem. A 3 (2015) 20218-20224.

[43] A. L. M. Reddy, A. Srivastava, S. R. Gowda, H. Gullapalli, M. Dubey, P. M. Ajayan, ACS Nano 4 (2010) 6337-6342.

[44] X. Wang, X. Cao, L. Bourgeois, H. Guan, S. Chen, Y. Zhong, D. M. Tang, H. Li, T. Zhai, L. Li, Adv. Funct. Mater. 22 (2012) 2682-2690.

[45] L. Qu, Y. Liu, J.-B. Baek, L. Dai, ACS Nano 4 (2010) 1321-1326.

[46] R. Lv, T. Cui, M. S. Jun, Q. Zhang, A. Cao, D. S. Su, Z. Zhang, S. H. Yoon, J. Miyawaki, I. Mochida, Adv. Funct. Mater. 21 (2011) 999-1006.

[47] S. Stankovich, D. A. Dikin, R. D. Piner, K. A. Kohlhaas, A. Kleinhammes, Y. Jia, Y. Wu, S. T. Nguyen, R. S. Ruoff, Carbon 45 (2007) 1558-1565.

[48] J. Song, M. L. Gordin, T. Xu, S. Chen, Z. Yu, H. Sohn, J. Lu, Y. Ren, Y. Duan, D. Wang, Angew. Chem. Int. Ed. 54 (2015) 4325-4329.

[49] N. McIntyre, D. Zetaruk, Anal. Chem. 49 (1977) 1521-1529. 
[50] G. Panzmer, B. Egert, Surf. Sci. 144 (1984) 651-664.

[51] C. Dong, X. Zheng, B. Huang, M. Lu, Appl. Surf. Sci. 265 (2013) 114-119.

[52] Y. X. Yin, S. Xin, Y. G. Guo, L. J. Wan, Angew. Chem. Int. Ed. 52 (2013) 13186-13200.

[53] N. Jayaprakash, J. Shen, S. S. Moganty, A. Corona, L. A. Archer, Angew. Chem. Int. Ed. 50 (2011) 5904-5908.

[54] M. Q. Zhao, Q. Zhang, J. Q. Huang, G. L. Tian, J. Q. Nie, H. J. Peng, F. Wei, Nat. Commun. 5 (2014) 3410.

[55] V. S. Kolosnitsyn, E. V. Karaseva, Russ. J. Electrochem. 44 (2008) 506-509.

[56] G. Babu, N. Masurkar, H. Al Salem, L. M. R. Arava, J. Am. Chem. Soc. 139 (2017) 171178.

[57] L. Li, L. Chen, S. Mukherjee, J. Gao, H. Sun, Z. Liu, X. Ma, T. Gupta, C. V. Singh, W. Ren, H.-M. Cheng, N. Koratkar, Adv. Mater. 29 (2017) 1602734.

[58] H. Al Salem, G. Babu, C. V. Rao, L. M. R. Arava, J. Am. Chem. Soc. 137 (2015) 1154211545. 